\title{
Desempenho organizacional de seguradoras de vida e previdência no Brasil: uma análise apoiada em DEA
}

\author{
Marcelo Alvaro da Silva Macedo \\ Doutorado em Engenharia de Produção pela Universidade Federal do Rio de Janeiro - \\ UFRJ \\ Professor da Universidade Federal Rural do Rio de Janeiro - UFRJ \\ Rod. BR 465, Km 07. Seropédica/RJ. CEP: 23890-000 \\ E-mail: alvaro@ufrrj.br \\ Ana Carolina Thomaz de Almeida Monteiro Barbosa \\ Graduando em Administração na Universidade Federal do Rio de Janeiro - FRRJ \\ Bolsista na Universidade Federal Rural do Rio de Janeiro - UFRRJ \\ Rod. BR 465, Km 07. UFRRJ. Seropédica/RJ. CEP: 23890-000 \\ E-mail: acbarbosa@ufrrj.br
}

\section{RESUMO}

A avaliação de desempenho é algo importante na gestão de empresas de qualquer setor de atuação, pois, desta forma a administração da organização se torna capaz de monitorar, comparar e, até mesmo, corrigir o desempenho da mesma. Sendo assim, a avaliação de eficiência é fundamental para a sobrevivência da organização num ambiente competitivo. Neste sentido, este trabalho procura, através da metodologia DEA (Análise Envoltória de Dados), com retornos constantes de escala (CRS) e orientações input e output, analisar o desempenho das seguradoras de vida e previdência no ano de 2005, mediante três modelagens. Na primeira, busca-se eficiência de cada instituição através da análise dos indicadores de entrada (inputs) gasto com benefício e sinistralidade, para obtenção de resultado financeiro (output). Na segunda, busca-se eficiência através da análise dos mesmos indicadores de entrada para a obtenção de margem de lucro (output). Por fim, a última combina todos os inputs e outputs. Os resultados mostraram que as seguradoras com melhor desempenho não são necessariamente de grande porte e nem estão ligadas a grandes conglomerados financeiros.

Palavras-chave: Desempenho Organizacional. Seguradoras de Vida e Previdência. DEA.

\section{Organizational Performance of Brazilian Life and Providence Insurance Companies': A DEA Approach}

\section{ABSTRACT}

The performance evaluation is something important in management of all sectors. Doing this, in this sense, the organization's manager become capable to monitor, compare and, even though, correct the performance of the same one. In this sense, the efficiency measurement is the base for the organization survival in a competitive environment. In this 
Marcelo Alvaro da Silva Macedo, Ana Carolina Thomaz de Almeida Monteiro Barbosa

sense, this study looks for, through DEA (Data Envelopment Analysis) methodology, using constant returns of scale (CRS) and output and input orientations, to analyze the performance of life and providence insurance companies in 2005 using three models. The first model searches for efficiency of each institution through the analysis of the inputs indicators: Benefits and Claim Ratios to obtain Financial Result (output). The second one searches for efficiency through the analysis of the same inputs to obtain Operational Profitability (output). Finally, the last one combines all inputs and outputs. The results show that companies with better performance are not, necessarily, the biggest one and nor related to big financial groups.

Keywords: Organizational Performance. Life and Providence Insurance Companies. DEA.

\section{INTRODUÇÃO}

Segundo Yang (2006), de maneira geral, no mundo, o mercado de seguros tem caminhado no sentido de impor às seguradoras uma mudança de suas ações estratégicas a fim de reduzir custos, com a manutenção ou melhoria da qualidade dos serviços prestados. Então, tornam- se imprescindíveis para os gestores, ferramentas que possam mostrar o posicionamento de suas organizações em relação aos competidores, no que diz respeito à capacidade de responder e se adaptar ao ambiente em transformação. Neste sentido, a avaliação do desempenho se torna um poderoso mecanismo de gestão.

Não existe um modelo de avaliação de desempenho organizacional que seja único para abarcar todas as variáveis do mundo empresarial. No entanto, alguns métodos são capazes de considerar aspectos diversificados, assumindo, assim, um papel crucial na avaliação da performance empresarial, como a medida de eficiência da Análise Envoltória de Dados (DEA), utilizada neste trabalho.

Para Macedo et al., (2006), a resposta mais importante desta metodologia é a caracterização de uma medida de eficiência, que faz com que a decisão fique orientada por um único indicador construído a partir de várias abordagens de desempenho diferentes. Vale ressaltar que isso facilita o processo decisório, pois, ao invés de considerar vários índices para concluir a respeito do desempenho da empresa ou da unidade sob análise, o gestor utiliza apenas a medida de eficiência da DEA. Além disso, a metodologia DEA mune a alta administração com informações 
Marcelo Alvaro da Silva Macedo, Ana Carolina Thomaz de Almeida Monteiro Barbosa

adicionais sobre os maiores determinantes de eficiência ou ineficiência, partindo-se de variáveis pré-selecionadas, através uma análise de benchmarking, com a qual o gestor pode avaliar as alterações necessárias para que a entidade possa se tornar eficiente em termos competitivos.

É neste contexto que se insere o trabalho, aplicando DEA na avaliação do desempenho das maiores seguradoras de vida e previdência em atividade no Brasil, no ano de 2005. Utilizando três modelos construídos a partir das variáveis de entrada: gasto com benefício; e sinistralidade e saída; margem e resultado financeiro, busca-se discutir a performance multicriterial relativa de 15 seguradoras de vida e previdência dentre as listadas como as maiores e melhores do setor pela Revista Valor Financeiro, na edição de 2006.

\section{DESEMPENHO ORGANIZACIONAL}

A globalização e a abertura dos mercados fizeram com que as organizações sofressem mudanças para se adaptarem ao novo quadro mundial. Neste quadro, em que a competitividade é um fator determinante, os sistemas de medição de desempenho se tornaram indispensáveis.

A mensuração de desempenho é definida por Neely (1998) como um processo de quantificação da eficiência e da efetividade de ações passadas, mediante a aquisição, coleta, classificação, análise, interpretação e divulgação dos dados apropriados. Ao final desta trajetória, os sistemas de medição fornecem o grau de evolução, estagnação ou involução dos processos e atividades das empresas sob análise, gerando informações importantes e a tempo de que as ações preventivas e/ou corretivas sejam tomadas. Como o progresso da empresa é monitorado através destes sistemas, seus eventuais erros são corrigidos, aumentando as possibilidades de que estas conquistem suas metas e objetivos.

Os processos de mensuração de desempenho se desenvolvem a partir da seleção de indicadores críticos de desempenho específicos para cada empresa. Entretanto, a maior dificuldade dos gestores está na escolha desses indicadores. O principal meio de definir estes indicadores seria, primeiramente, definir onde a organização se encontra e 
Marcelo Alvaro da Silva Macedo, Ana Carolina Thomaz de Almeida Monteiro Barbosa

aonde quer chegar, para posteriormente definir os mecanismos que farão com que isso seja possível. Tais processos são permanentes e repetitivos, sendo a frequência de suas medições dependente da atividade a ser medida.

Para Slack et al., (2002), toda organização precisa, de alguma forma, de medidas de desempenho como um pré-requisito para melhoramento. No entanto, as práticas de medição de desempenho variam entre as empresas. Cabe ressaltar, porém, que depois da mensuração de desempenho, usando, normalmente, um conjunto de medidas parciais, a organização precisa fazer um julgamento sobre sua performance. Este julgamento, segundo os autores, envolve a comparação do nível de desempenho atingido com algum tipo de padrão. Esses padrões podem ser:

- Padrões Históricos: consistem na comparação do desempenho atual com desempenhos anteriores, construindo assim uma tendência do comportamento de desempenho ao longo do tempo.

- Padrões de Desempenho Alvos: são aqueles estabelecidos arbitrariamente para refletir algum nível de desempenho que é visto como adequado.

- Padrões de Desempenho da Concorrência: utilizam a comparação do desempenho atingido com aquele que está sendo conseguido por um ou mais competidores.

- Padrões de Desempenho Absolutos: são aqueles tomados em seus limites (ex.: estoque zero, zero defeito, etc.).

Quando as empresas utilizam o mercado como parâmetro de comparação, segundo padrões de desempenho da concorrência, é necessário que se use uma abordagem denominada benchmarking. Essa abordagem consiste na performance relativa das empresas em função de um conjunto de competidores previamente selecionados (Slack et al., 2002).

Camp (1989) define benchmarking como sendo um processo sistemático para a identificação da melhor prática e para a modificação do conhecimento existente, de modo a alcançar um desempenho superior. Para Novaes (2004), benchmarking é considerado um processo contínuo e sistemático de empresas e serviços, através da sua comparação 
Marcelo Alvaro da Silva Macedo, Ana Carolina Thomaz de Almeida Monteiro Barbosa

com unidades consideradas eficientes, levando ao estabelecimento de ações gerenciais efetivas, com o objetivo de aprimorar os resultados (redução de custos, aumento de produção, etc.); além de ser um dos modernos instrumentos de gerência, que possibilita a melhoria do desempenho técnico-econômico das empresas de forma comparativa ou relativa.

Os sistemas de mensuração de desempenho são essenciais para que as organizações possam competir no mercado global. Porém, as avaliações feitas em caráter relativo, ou seja, mensurando a eficiência da organização em relação, por exemplo, ao seu ambiente competitivo, são geradoras de resultados potencialmente consistentes. Isto ocorre à medida que informam o posicionamento da empresa perante a referência e às demais empresas.

Não existe possibilidade de reduzir a complexidade do desempenho de um negócio a um único indicador, havendo necessidade real de aplicar diversos indicadores para atingir os mais variados aspectos nos quais a estratégia de negócio se realiza (Slack et al., 2002). Assim, um programa de avaliação de desempenho eficiente deve incluir todos esses elementos considerados críticos de sucesso. Isto quer dizer que todos os elementos relevantes às empresas, de maneira individual, precisam ser, de alguma forma, mensurados para permitir análises comparativas.

São inúmeras as variáveis do mundo empresarial passíveis de mensuração. No entanto não existem, ainda, modelos de avaliação de performance únicos para todas estas variáveis. A solução encontrada pelos gestores é a utilização de uma série de metodologias de avaliação capazes de tratar os diferentes elementos da organização. Essas técnicas produzem resultados isolados, uma vez que não consideraram a multiplicidade de fatores numa única análise.

Neste sentido, a DEA, apresenta-se como uma medida de desempenho capaz de comparar a eficiência de várias unidades operacionais similares mediante a consideração explícita do uso de suas múltiplas entradas para a produção de múltiplas saídas. Desta forma, esta metodologia faz com que a decisão fique orientada por um único indicador construído a partir de várias abordagens de desempenho diferentes. Portanto, como congrega diversas perspectivas, a técnica promove uma melhor percepção da 
Marcelo Alvaro da Silva Macedo, Ana Carolina Thomaz de Almeida Monteiro Barbosa performance organizacional.

Segundo Silva; Neves e Gonçalves Neto (2003), a metodologia DEA, que teve origem no trabalho de Farrell (1957), caracteriza-se como uma técnica não paramétrica que permite lidar com várias saídas (outputs) e entradas (inputs), com o objetivo de analisar, comparativamente, unidades independentes no que se refere ao seu desempenho, ou seja, à eficiência de cada unidade.

De acordo com Yang (2006), a DEA se propõe a analisar a relação recursos/produção, ou, ainda, entradas/saídas, envolvida na avaliação do desempenho de unidades organizacionais, indicando os fatores que interferem positiva ou negativamente na eficiência destas. Sendo assim, um dos maiores benefícios do uso da DEA é o conjunto de unidades de referência que pode ser usado como benchmarking na melhoria do desempenho das unidades menos eficientes. Estes benchmarks indicam o que precisa ser modificado nos inputs e outputs e como melhorá-los para transformar unidades ineficientes em eficientes.

DEA, segundo Zhu (2000), representa uma das mais adequadas ferramentas para avaliar a eficiência, em comparação com ferramentas convencionais. Os resultados da DEA são mais detalhados do que os obtidos em outras abordagens, servindo melhor ao embasamento de recomendações de natureza gerencial. Sendo assim, os autores destacam as seguintes características desta ferramenta:

- Não requer a priori uma função de produção explícita;

- Examina a possibilidade de diferentes, mas igualmente eficientes combinações de

- Inputs e outputs;

- Localiza a fronteira eficiente dentro de um grupo de unidades analisadas;e

- Idetermina, para cada unidade ineficiente, subgrupos de unidades eficientes, os quais formam seu conjunto de referência.

São várias as formulações dos modelos de DEA encontrados na literatura, conforme mencionam Charnes et al., (1994), entretanto, dois modelos básicos DEA são geralmente usados nas aplicações. O primeiro modelo chamado de CCR (Charnes; 
Marcelo Alvaro da Silva Macedo, Ana Carolina Thomaz de Almeida Monteiro Barbosa

Cooper; Rhodes, 1978), também conhecido como CRS (Constant Returns to Scale), avalia a eficiência total, identifica as DMUs eficientes e ineficientes e determina a que distância da fronteira de eficiência estão as unidades ineficientes. O segundo, chamado de modelo BCC (Banker, Charnes; Cooper, 1984), também conhecido como VRS (Variable Returns to Scale), utiliza uma formulação que permite a projeção de cada Decision Making Unit (DMU) ineficiente sobre a superfície de fronteira (envoltória) determinada pelas DMUs eficientes de tamanho compatível.

No caso das formulações, além da escolha entre CRS e VRS existe a necessidade de fixação da ótica de análise (orientação input ou orientação output). Macedo et al., (2006) assinalou que a abordagem DEA baseada nas entradas (inputs) busca maximizar as quantidades de produtos, isto é, maximizar uma combinação linear das quantidades dos vários produtos da empresa. Já para uma abordagem baseada nas saídas (outputs), busca-se minimizar as quantidades de insumos, isto é, minimizar uma combinação linear das quantidades dos vários insumos da empresa.

De acordo com Silva e Neves (2004), dentre as propriedades que levaram esta técnica a ser adotada como método de análise de vários estudos, pode-se destacar as seguintes:

- Difere dos métodos que necessitam que todos os inputs e outputs sejam transformados em uma única unidade de medida;

- É uma alternativa aos métodos tradicionais de análise de tendência central e custo-benefício;

- Ser possíveis benchmarks para serem estudados pelas demais unidades (aprender com os melhores daquele segmento);

- Identificação das dimensões da ineficiência relativa de cada uma das unidades comparadas, bem como do índice de eficiência de cada uma;

- É baseado em informações individuais de cada unidade, também chamada de DMU (Decision Making Unit), sendo possível utilizar múltiplos outputs e múltiplos inputs, além da possibilidade das variáveis estarem em unidades completamente diferentes; 
Desempenho organizacional de seguradoras de vida e previdência no Brasil: uma análise apoiada

em DEA

Marcelo Alvaro da Silva Macedo, Ana Carolina Thomaz de Almeida Monteiro Barbosa

- Permite que sejam identificadas qualidades comuns entre os mais/menos eficientes.

\section{DESEMPENHO ORGANIZACIONAL NO RAMO DE SEGUROS}

A primeira fase de expansão dos seguros no Brasil ocorreu na década 1970, logo após a reestruturação do sistema financeiro nacional. Um outro salto aconteceu com a implantação do Plano Real, que fez com que houvesse um acréscimo na renda dos brasileiros, impulsionando o mercado de seguros.

Entre os anos de 1995 e 2003 o mercado teve um crescimento equivalente a $188 \%$. No entanto, nesta época, o Brasil continuava apresentando índices baixos de consumo de seguros quando comparado a outros países em desenvolvimento. $O$ mercado continuou a crescer em 2004 e 2005, embora em taxas menores às observadas na décadaanterior.

Esse baixo crescimento acompanhado da redução das taxas de juros fez com que o setor passasse por uma pequena crise. Neste ponto, cabe ressaltar que um dos motivos para as seguradoras conseguirem fechar com êxito seus balanços são os ganhos garantidos não pela atividade principal destas, mas pela aplicação de reservas no mercado financeiro. São as altas taxas de juros características do país as responsáveis por tirar as seguradoras do déficit.

Em 2004, uma queda na taxa de juros provocou consideráveis perdas nos ganhos das seguradoras, que mesmo assim conseguiram, através destes, assegurar lucro no final. Para enfrentar a conjuntura não muito favorável, as seguradoras cortaram custos e ofereceram novos serviços (populares) que ajudaram a ampliar a base de consumidores. Em 2005, a elevação da taxa de juros favoreceu novamente os ganhos financeiros, impedindo que os balanços fossem negativos.

Silva; Neves e Gonçalves Neto (2003) esclarecem que o mercado de seguros no Brasil possui um incrível potencial de crescimento. Já Contador et al., (2000) ressaltam que a concorrência, nessa "indústria", se apresenta em transformação, em que as mudanças no mercado ocasionam mudanças também nas empresas do setor. As altas 
Marcelo Alvaro da Silva Macedo, Ana Carolina Thomaz de Almeida Monteiro Barbosa

taxas de inflação tornam menos rigorosa a seleção de riscos. Isto ocorre porque as aplicações no mercado financeiro podem gerar lucros altíssimos. Porém, quando a inflação está em baixa, os ganhos financeiros não compensam prejuízos decorrentes da má seleção de riscos.

Contador et al., (2000), em seu artigo, tratam da avaliação de seguradoras que atuam no Brasil, procurando destacar a maior eficiência em subscrição de riscos, aplicando para isso o método da DEA.

Para colocar em prática os conceitos, o artigo baseou-se no Boletim Estatístico da Superintendência de Seguros Privados (SUSEP) do primeiro semestre de 1999 para selecionar um grupo de 52 seguradoras com vendas superiores a $R \$ 5$ milhões no período. As variáveis escolhidas como inputs foram: sinistro retido e despesa comercial e como output, o prêmio ganho.

É importante destacar que a orientação utilizada foi à maximização de output e a escala a VRS (Retorno Variável de Escala). Como resultado, os autores constataram que somente 10 empresas estudadas eram eficientes; dessas dez, cinco pertenciam a conglomerados bancários, que dispõem de uma boa rede de distribuição dos produtos (agências bancárias), deixando claro a dominação da venda casada.

Alguns outros trabalhos demonstram também a relevância da ligação entre seguradoras e conglomerados financeiros. Neles, percebe-se que a eficiência máxima está relacionada à essa associação.

Silva; Neves e Gonçalves Neto (2003), em seu artigo, avaliam o setor segurador brasileiro, através de um estudo comparativo entre as maiores seguradoras do país no ano de 2002, empregando a técnica da DEA, que permite estabelecer um benchmark de eficiência.

Para isso, foram escolhidas as 11 maiores seguradoras (em termos de Patrimônio Líquido), de acordo com o descrito junto ao Boletim Estatístico da SUSEP 2002 (período de Janeiro a Novembro de 2002). O Modelo DEA-BCC, com orientação à maximização dos outputs, foi o escolhido para calcular os níveis de eficiência relativa.

As seguintes variáveis foram selecionadas: despesas comerciais, despesas administrativas, prêmios de seguros e índices de sinistralidade. As duas primeiras como 
Marcelo Alvaro da Silva Macedo, Ana Carolina Thomaz de Almeida Monteiro Barbosa inputs e as duas últimas como outputs.

Antes de realizar a modelagem com os dados foram retiradas 3 unidades das 11 escolhidas, por conta de problemas nos dados apresentados. O resultado apresentou duas unidades com eficiência máxima (100\%). Além disso, foi percebido que das oito empresas testadas, as cinco mais eficientes eram pertencentes a conglomerados financeiros. Isto mostra a existência do ganho de escala para essas empresas no segmento de seguro, já que utilizam o canal de distribuição das agências para vender seus produtos.

Silva e Neves (2004) analisaram a estratégia empregada no canal de distribuição, no tocante às seguradoras de grande e médio porte no mercado brasil.

Os autores estimaram a eficiência de cada seguradora em relação às demais do segmento, utilizando uma orientação output e o modelo CRS. Numa análise geral dos vários segmentos de seguro, mostraram que os grandes conglomerados financeiros não conseguem necessariamente obter maiores desempenhos, contrariando as pesquisas anteriores. Isto mostra que para competir neste mercado pode ser necessário um foco operacional cada vez maior, trazendo para o setor uma nova perspectiva competitiva, pois os ganhos financeiros que estes grandes conglomerados poderiam obter se mostram cada vez menos eficientes, passando assim a competição a ser centrada numa disputa operacional, focada, por exemplo, numa gestão eficiente do risco (sinistralidade). Eles concluem que instituições de pequeno e médio porte possuem totais condições de sobrevivência e de competição neste setor, nos diversos segmentos, ressaltando o fato de que não é o tamanho que gera a competitividade, mas sim o desempenho superior.

Arrieta e Rieckhof (2001) fizeram um estudo que compara a eficiência técnica e de escala entre 20 seguradoras de vida do Perú, divididas em especializadas e mistas. Utilizando um modelo DEA-CRS e DEA-VRS e tendo como variáveis de input gastos operacionais, patrimônio líquido e ativo total e como outputs, vendas líquidas e margem de lucro, os autores concluíram que na eficiência técnica encontra-se um resultado melhor para as seguradoras especializadas. Porém, na eficiência de escala encontra-se um resultado melhor nas seguradoras mistas.

Na pesquisa de Hsiao e Su (2006), o objetivo era determinar a eficiência do capital 
Marcelo Alvaro da Silva Macedo, Ana Carolina Thomaz de Almeida Monteiro Barbosa

investido utilizando DEA, verificando se existe diferença entre 25 seguradoras de vida de Taiwan divididas em três grupos: seguradoras nacionais antigas, seguradoras nacionais novas e seguradoras estrangeiras. Os resultados não mostram diferenças significativas entre os três grupos. Utilizando a eficiência global (CRS), técnica (VRS) e de escala, os autores concluíram que não existem diferenças significativas entre os desempenhos dos três grupos.

Yang (2006) avaliou, em seu trabalho, os impactos do desempenho de 72 seguradoras de Vida e Saúde do Canadá, sobre as questões operacionais e estratégicas durante o ano de 1998. Através de um modelo de dois estágios, o autor integrou uma avaliação com foco na produção com outra com foco nos investimentos. Por intermédio de um modelo com retornos variáveis e constantes de escala e, utilizando variáveis tais como mão-de-obra, outros gastos operacionais, prêmio ganho, provisões técnicas, total dos investimentos, dentre outros, o autor concluiu que a modelagem DEA é capaz de prover os gestores com informações sobre como se encontra suas organizações e como melhorar este desempenho frente ao ambiente competitivo.

\section{METODOLOGIA}

Esta pesquisa pode ser caracterizada, de acordo com o exposto por Vergara (2007), como sendo descritiva e quantitativa, pois procura-se através da aplicação da análise envoltória de dados às informações das seguradoras que fazem parte da amostra, expor características a respeito da sua performance.

O processo de amostragem é não probabilístico, pois parte-se de um universo naturalmente restrito, já que as seguradoras foram escolhidas a partir das que constavam na publicação utilizada. Isso traz algumas limitações de inferência, mas não invalida os resultados.

- Gasto com benefício (GAS): Representa o input 01 e é obtido pela divisão de despesas com benefícios e resgates pelas rendas de contribuições líquidas. É um indicador específico da atividade de previdência. Indica o quanto o uso de 
Marcelo Alvaro da Silva Macedo, Ana Carolina Thomaz de Almeida Monteiro Barbosa benefícios representa do total de receitas previdenciárias. É um índice do tipo quanto menor, melhor.

- Sinistralidade (SIN): Representa o input 02 e é obtido pela divisão de sinistros retidos pelos prêmios ganhos. Mede quanto o montante de sinistros representa do total de receita líquida de prêmios, indicando o quanto de suas contribuições os beneficiários de seguros de vida estão utilizando. É um índice do tipo quanto menor, melhor o desempenho.

- Resultado financeiro (RES): Representa o output 01 e é obtido pela divisão do resultado financeiro pelas aplicações. É um índice do tipo quanto maior, melhor.

- Margem (MAR): Representa o output 02 e é obtido pela divisão do resultado operacional pela soma das rendas de contribuições líquidas (provenientes da atividade previdência) com os prêmios ganhos (proveniente da atividade vida). É um índice do tipo quanto maior, melhor.

Estas variáveis foram escolhidas por uma série de motivos. Os inputs, por representarem o sacrifício em cada uma das operações, vida e previdência. Os outputs, por representarem ganhos financeiros e operacionais das seguradoras. Especificamente os ganhos financeiros estão destacados em virtude de vários estudos apontarem para este fator como sendo o grande formador de lucratividade no ramo de seguros.

Um grande número de diferentes variáveis, como as apresentadas anteriormente, pode ser utilizado para avaliar a eficiência de unidades organizacionais, tais como seguradoras onde cada uma destas pode ser vista como um vetor de desempenho que representa um diferente aspecto da performance. Estes seriam modelos de avaliação de performance monocriteriais.

O que se busca neste artigo é apresentar uma metodologia multidimensional, na qual seja possível avaliar o desempenho de cada seguradora de vida e previdência de modo multicriterial, ou seja, considerando de maneira integrada todos os vetores de desempenho apresentados. Além disso, busca-se uma visão ao longo do tempo, para evitar oscilações esporádicas e pontuais.

Isto é feito através da utilização da DEA, que mostra o quão uma seguradora é 
eficiente no tratamento de seus inputs e outputs, em relação às demais. Esta análise fornece um indicador que varia de 0 a 1 ou de $0 \%$ a $100 \%$, sendo que somente as seguradoras que obtêm índice de eficiência igual a um é que são efetivamente eficientes, ou seja, fazem parte da fronteira eficiente. Em termos práticos, o modelo procura identificar a eficiência de uma seguradora comparando-a com os melhores desempenhos observados em seu nível de operação em cada ano.

Lins e Meza (2000) ressaltam que um caminho intuitivo para introduzir DEA é por meio de forma de razão. Para cada DMU, procura-se obter uma medida de razão de todos os outputs sobre todos os inputs ou ainda de todos os inputs sobre todos os outputs. Ou seja, a modelagem procura encontrar os pesos ótimos uj e vi para a resolução de um dos seguintes problemas de programação matemática:

$$
\begin{aligned}
& \operatorname{Max} E_{c}=\frac{\sum_{j=1}^{s} u_{j} y_{j c}}{\sum_{i=1}^{m} v_{i} x_{i c}} \\
& \text { S.a.: } \quad \frac{\sum_{j=1}^{s} u_{j} y_{j k}}{m} \leq 1, k=1,2, \ldots, c, \ldots, n \\
& \sum_{i=1} v_{i} x_{i k} \\
& u_{j} \geq 0, \forall j \text {, } \\
& v_{i} \geq 0, \forall i \\
& \text { Orientação input } \\
& \begin{array}{rl|}
\hline \text { Orientação output } \\
\text { Min } E_{c}=\frac{\sum_{i=1}^{m} v_{i} x_{i c}}{\sum_{j}^{s} u_{j} y_{j c}} \\
\text { S.a.: } \quad \frac{\sum_{i=1}^{m} v_{i} x_{i k}}{s} \geq 1, k=1,2, \mathrm{~K}, c, \mathrm{~K}, n \\
& \sum_{j=1}^{s} u_{j} y_{j k} \\
& u_{j} \geq 0, \forall j, \\
& v_{i} \geq 0, \forall i
\end{array}
\end{aligned}
$$

No modelo com orientação input, c é a unidade (DMU) que está sendo avaliada. O problema acima envolve a procura de valores para u e $v$, que são os pesos, de modo que maximize a soma ponderada dos outputs (output "virtual") dividida pela soma ponderada dos inputs (input "virtual") da DMU em estudo, sujeita a restrição de que esse quociente seja menor ou igual a um para todas as DMUs. Esta função está sujeita à restrição de que, quando o mesmo conjunto de coeficientes de entrada e saída (os vários vi e uj) for aplicado a todas as outras unidades de serviços que estão sendo comparadas, nenhuma 
unidade excederá $100 \%$ de eficiência ou uma razão de 1,00.

Já no modelo com orientação output, a eficiência é calculada pelo inverso da função objetivo

ou seja, eficiência $=1$ E . Este problema define a relação dos inputs sobre os outputs, onde c é o índice da unidade que está sendo avaliada. Tem-se neste problema as mesmas variáveis de decisão ux e vy, porém se quer minimizar a soma ponderada dos inputs ("input" virtual) dividida pela soma ponderada dos outputs ("output" virtual) da DMU em estudo, sujeita a restrição de que este quociente seja maior ou igual a 1, para todas as DMUs.

Segundo Coelli et al., (1998), este é um problema fracionário (não linear) de programação matemática de difícil solução, que pode ser facilmente resolvido transformando a relação em uma função linear, simplesmente considerando o denominador da função objetivo igual a um. De acordo com Charnes et al., (1994), os modelos DEA-CRS-I e DEA-CRS-O podem, então, ser apresentados da seguinte maneira:

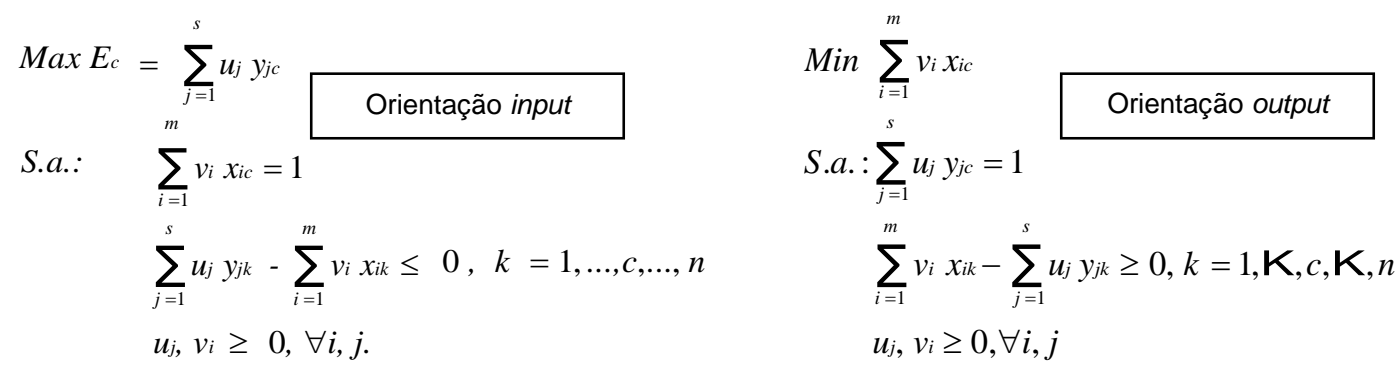


Estes modelos (DEA-CRS-I e DEA-CRS-O) são utilizados para a análise dos dados para que se tenha, ao mesmo tempo, duas óticas: melhorias dos inputs e melhorias dos outputs.

Utilizou-se três modelos de DEA para análise das seguradoras. O primeiro, com ênfase no desempenho financeiro, combinava os inputs 01 e 02 com o output 01 , verificando a eficiência através da análise da combinação entre os indicadores de gasto com benefícios e sinistralidade para obtenção de resultado financeiro. O segundo, com ênfase operacional, combinava os inputs 01 e 02 com o output 02 , verificando a eficiência através da análise da combinação dos mesmos indicadores de entrada para a obtenção de margem de lucro. Por último, um modelo completo combinava todos os inputs e outputs. Os dados utilizados na análise podem ser vistos a seguir, apresentados por seguradora:

Tabela 1 - Variáveis de Análise por Seguradora

\begin{tabular}{|l|c|c|c|c|}
\hline \multirow{2}{*}{ Companhia } & \multicolumn{2}{c|}{ Inputs } & \multicolumn{2}{c|}{ Outputs } \\
\cline { 2 - 5 } & GAS & SIN & RES & MAR \\
\hline Bradesco Vida e Previdência & 0,924 & 0,637 & 0,032 & 0,470 \\
\hline Itaú Vida & 0,960 & 0,280 & 0,013 & 0,788 \\
\hline Unibanco AIG Vida e Previdência & 1,014 & 0,361 & 0,011 & 0,174 \\
\hline Santander Seguros & 2,232 & 1,161 & 0,127 & 0,508 \\
\hline HSBC Vida e Previdência & 0,857 & 0,408 & 0,003 & 0,067 \\
\hline Icatu Hartford & 0,956 & 0,552 & 0,025 & 0,109 \\
\hline Porto Seguro Vida e Previdência & 0,999 & 0,648 & 0,040 & 0,177 \\
\hline Safra Vida e Previdência & 1,421 & 0,205 & 0,052 & 0,584 \\
\hline Mapfre Vera Cruz Vida e Prev. & 0,952 & 0,503 & 0,041 & 0,006 \\
\hline Sudameris Vida e Previdência & 0,998 & 0,241 & 0,047 & 0,169 \\
\hline Unimed & 0,988 & 0,604 & 0,031 & 0,170 \\
\hline MetLife Vida e Previdência & 0,988 & 0,287 & 0,052 & 0,061 \\
\hline Alfa Previdência Vida & 1,000 & 0,419 & 0,018 & 0,039 \\
\hline Mongeral & 0,263 & 0,495 & 0,095 & 0,035 \\
\hline Previsul & 1,200 & 0,529 & 0,124 & 0,055 \\
\hline
\end{tabular}

Estes dados revelam que das 15 seguradoras analisadas, 13 seguradoras apresentam uma taxa de uso superior a 90 \% em relação à operação de previdência. Destas, quatro chegam a apresentar este indicador acima de 1,00. Isso significa que 
Marcelo Alvaro da Silva Macedo, Ana Carolina Thomaz de Almeida Monteiro Barbosa

nesta operação o uso (gastos com benefícios) praticamente se iguala ou supera a arrecadação (rendas de contribuições líquidas), que quer dizer que na atividade de previdência a operação é deficitária na quase totalidade das seguradoras da amostra.

\section{APRESENTAÇÃO E ANÁLISE DOS RESULTADOS}

Com base nas informações das seguradoras de vida e previdência sob análise, montou-se os modelos de avaliação de eficiência para o ano de 2005. Em todas as análises utilizou-se modelos DEA-CRS com orientação input. Porém, no modelo considerando todas as variáveis, utilizou-se também a ótica dos outputs.

Para obter-se a eficiência de cada DMU utilizou-se um software de DEA, apresentado por Meza et al., (2003) denominado SIAD (Sistema Integrado de Apoio à Decisão). Além disso, analisou-se as mudanças nos níveis de inputs ou nos níveis de outputs nas seguradoras ineficientes, para que as mesmas se tornem eficientes. Cabe ressaltar, que a análise é feita sob duas óticas, ou seja, de melhorias/redução nos inputs ou por melhorias/incremento dos outputs. É claro que a seguradora pode adotar um caminho híbrido que considere os dois benchmarks apontados pela metodologia.

Com base nas informações da Tabela 2 pode-se verificar, quanto ao desempenho, que três (Safra, Mongeral e Previsul) das quinze seguradoras foram eficientes no modelo com o resultado financeiro como output. Todas as demais seguradoras tiveram desempenho muito longe do ótimo, sendo que a mais próxima tinha indicador apenas de 80,54\% (Sudameris). Na verdade, estas três seguradoras eficientes foram as que melhor combinaram os inputs (menores) e output (maior). Assim sendo, não precisam melhorar em nada seu desempenho.

Já as seguradoras HSBC, Unibanco, Alfa, Itaú e Icatú, que possuíam índices de eficiência baixíssimos, necessitam de mudanças radicais. Estes resultados negativos destas seguradoras se devem basicamente ao fato destas terem ganhos financeiros muito baixos em relação a amostra no ano de 2005. 
Desempenho organizacional de seguradoras de vida e previdência no Brasil: uma análise apoiada

Marcelo Alvaro da Silva Macedo, Ana Carolina Thomaz de Almeida Monteiro Barbosa

Tabela 2 - Eficiência e Valores Ideais para Inputs com RES como Output

\begin{tabular}{|c|c|c|c|c|c|}
\hline \multirow[b]{2}{*}{ Companhia } & \multirow[b]{2}{*}{ EFF } & \multicolumn{2}{|c|}{ Inputs Ideais } & \multicolumn{2}{|c|}{$\%$ de Melhoria } \\
\hline & & GAS & SIN & GAS & SIN \\
\hline Bradesco Vida e Previdência & 0,2343 & 0,2165 & 0,1493 & $76,57 \%$ & $76,56 \%$ \\
\hline Itaú Vida & 0,1941 & 0,1863 & 0,0543 & $80,58 \%$ & $80,60 \%$ \\
\hline Unibanco AIG Vida e Previdência & 0,1288 & 0,1306 & 0,0465 & $87,12 \%$ & $87,13 \%$ \\
\hline Santander Seguros & 0,4841 & 1,0806 & 0,5621 & $51,58 \%$ & $51,57 \%$ \\
\hline HSBC Vida e Previdência & 0,0319 & 0,0274 & 0,0130 & $96,81 \%$ & $96,81 \%$ \\
\hline Icatu Hartford & 0,2047 & 0,1957 & 0,1130 & $79,53 \%$ & $79,53 \%$ \\
\hline Porto Seguro Vida e Previdência & 0,2849 & 0,2847 & 0,1846 & $71,49 \%$ & $71,52 \%$ \\
\hline Safra Vida e Previdência & 1,0000 & 1,4210 & 0,2050 & \multicolumn{2}{|c|}{ EFICIENTE } \\
\hline Mapfre Vera Cruz Vida e Prev. & 0,3619 & 0,3446 & 0,1820 & $63,79 \%$ & $63,82 \%$ \\
\hline Sudameris Vida e Previdência & 0,8054 & 0,8038 & 0,1941 & $19,44 \%$ & $19,59 \%$ \\
\hline Unimed & 0,2344 & 0,2316 & 0,1416 & $76,55 \%$ & $76,58 \%$ \\
\hline MetLife Vida e Previdência & 0,7573 & 0,7482 & 0,2173 & $24,29 \%$ & $24,26 \%$ \\
\hline Alfa Previdência Vida & 0,1829 & 0,1829 & 0,0766 & $81,71 \%$ & $81,71 \%$ \\
\hline Mongeral & 1,0000 & 0,2630 & 0,4950 & \multicolumn{2}{|c|}{ EFICIENTE } \\
\hline Previsul & 1,0000 & 1,2000 & 0,5290 & \multicolumn{2}{|c|}{ EFICIENTE } \\
\hline
\end{tabular}

Tendo como base as informações da Tabela 3, pode-se verificar, quanto ao desempenho de margem operacional, que apenas a Itaú e a Safra foram eficientes. Todas as outras seguradoras tiveram desempenho muito longe do ótimo, sendo que a mais próxima tinha indicador apenas de 61,97 \% (Bradesco).

Já as seguradoras Mapfre, Alfa, Previsul, MetLife e HSBC, que apresentaram índice de eficiência baixíssimo, necessitam de mudanças radicais. Este fraco desempenho é justificado pela baixa margem de lucro que todas estas seguradoras têm em relação às demais. 
Marcelo Alvaro da Silva Macedo, Ana Carolina Thomaz de Almeida Monteiro Barbosa

Tabela 3 - Eficiência e Valores Ideais para Inputs com MAR como Output

\begin{tabular}{|l|c|c|c|c|c|}
\cline { 5 - 7 } \multicolumn{1}{c|}{} & \multicolumn{2}{c|}{ Inputs Ideais } & \multicolumn{2}{c|}{$\%$ de Melhoria } \\
\hline Bradesco Vida e Previdência & EFF & GAS & SIN & GAS & SIN \\
\hline Itaú Vida & 0,6197 & 0,5726 & 0,1670 & $38,03 \%$ & $73,78 \%$ \\
\hline Unibanco AIG Vida e Previdência & 1,0000 & 0,9600 & 0,2800 & \multicolumn{2}{c|}{ EFICIENTE } \\
\hline Santander Seguros & 0,2773 & 0,6189 & 0,1805 & $72,27 \%$ & $84,45 \%$ \\
\hline HSBC Vida e Previdência & 0,0952 & 0,0816 & 0,0238 & $90,47 \%$ & $94,17 \%$ \\
\hline Icatu Hartford & 0,1389 & 0,1328 & 0,0387 & $86,11 \%$ & $92,98 \%$ \\
\hline Porto Seguro Vida e Previdência & 0,2159 & 0,2156 & 0,0629 & $78,41 \%$ & $90,30 \%$ \\
\hline Safra Vida e Previdência & 1,0000 & 1,4210 & 0,2050 & \multicolumn{2}{|c|}{ EFICIENTE } \\
\hline Mapfre Vera Cruz Vida e Prev. & 0,0077 & 0,0073 & 0,0021 & $99,23 \%$ & $99,58 \%$ \\
\hline Sudameris Vida e Previdência & 0,2486 & 0,2481 & 0,0599 & $75,14 \%$ & $75,18 \%$ \\
\hline Unimed & 0,2096 & 0,2071 & 0,0604 & $79,03 \%$ & $90,01 \%$ \\
\hline MetLife Vida e Previdência & 0,0755 & 0,0746 & 0,0217 & $92,45 \%$ & $92,45 \%$ \\
\hline Alfa Previdência Vida & 0,0475 & 0,0475 & 0,0139 & $95,25 \%$ & $96,69 \%$ \\
\hline Mongeral & 0,1621 & 0,0426 & 0,0124 & $83,80 \%$ & $97,49 \%$ \\
\hline Previsul & 0,0558 & 0,0670 & 0,0195 & $94,42 \%$ & $96,31 \%$ \\
\hline
\end{tabular}

Numa análise com todas as variáveis (tabela 4), verificou-se que as seguradoras Itaú, Safra, Mongeral e Previsul foram eficientes. Na verdade, estas seguradoras foram as que obtiveram desempenho superior em um dos modelos anteriores. Isso acontece por conta da lógica do modelo de procurar maximizar a eficiência. Logo, se uma unidade é eficiente em pelo menos um dos outputs, ela será eficiente num modelo que considere os dois, pois a modelagem irá atribuir peso zero ao output em que a DMU tiver pior desempenho. Isso também explica o fato de todas as unidades terem eficiência no modelo completo igual ou superior a sua maior eficiência nos dois modelos anteriores. Esta é uma limitação da metodologia DEA, mas não chega a inviabilizar esta parte da análise, pois o objetivo é muito mais verificar as necessidades de melhorias nos inputs e outputs combinados, do que observar a eficiência.

A mesma lógica se aplica às piores seguradoras. Por conseguinte, Alfa e HSBC são as seguradoras com menor desempenho, pois são aquelas que aparecem com baixíssima eficiência em ambos os modelos anteriores. 


\section{Desempenho organizacional de seguradoras de vida e previdência no Brasil: uma análise apoiada \\ em DEA}

Marcelo Alvaro da Silva Macedo, Ana Carolina Thomaz de Almeida Monteiro Barbosa

Tabela 4 - Eficiência e Valores Ideais para Inputs e Outputs no Modelo Completo

\begin{tabular}{|l|c|c|c|c|c|c|c|c|c|}
\cline { 3 - 9 } \multicolumn{2}{c|}{} & \multicolumn{2}{c|}{ Inputs Ideais } & Outputs Ideais & \multicolumn{4}{c|}{$\%$ de Melhoria } \\
\hline Companhia & EFF & GAS & SIN & RES & MAR & GAS & SIN & RES & MAR \\
\hline Bradesco Vida e Previdência & 0,6809 & 0,6292 & 0,2909 & 0,0470 & 0,6902 & $31,91 \%$ & $54,32 \%$ & $46,60 \%$ & $46,82 \%$ \\
\hline Itaú Vida & 1,0000 & 0,9600 & 0,2800 & 0,0130 & 0,7880 & \multicolumn{3}{|c|}{ EFICIENTE } \\
\hline Unibanco AIG Vida e Previdência & 0,2570 & 0,2605 & 0,0928 & 0,0428 & 0,6772 & $74,30 \%$ & $74,31 \%$ & $299,53 \%$ & $289,38 \%$ \\
\hline Santander Seguros & 0,5649 & 1,2608 & 0,6558 & 0,2248 & 0,8993 & $43,51 \%$ & $43,50 \%$ & $77,22 \%$ & $77,00 \%$ \\
\hline HSBC Vida e Previdência & 0,1004 & 0,0860 & 0,0335 & 0,0299 & 0,6673 & $89,95 \%$ & $91,80 \%$ & $913,30 \%$ & $901,51 \%$ \\
\hline Icatu Hartford & 0,2507 & 0,2397 & 0,1384 & 0,0997 & 0,4348 & $74,93 \%$ & $74,92 \%$ & $303,01 \%$ & $299,83 \%$ \\
\hline Porto Seguro Vida e Previdência & 0,3585 & 0,3582 & 0,2323 & 0,1116 & 0,4937 & $64,13 \%$ & $64,16 \%$ & $181,63 \%$ & $179,66 \%$ \\
\hline Safra Vida e Previdência & 1,0000 & 1,4210 & 0,2050 & 0,0520 & 0,5840 & & EFICIENTE \\
\hline Mapfre Vera Cruz Vida e Prev. & 0,3619 & 0,3446 & 0,1820 & 0,1133 & 0,0487 & $63,79 \%$ & $63,82 \%$ & $178,41 \%$ & $684,16 \%$ \\
\hline Sudameris Vida e Previdência & 0,8054 & 0,8038 & 0,1941 & 0,0584 & 0,2907 & $19,44 \%$ & $19,59 \%$ & $23,66 \%$ & $71,97 \%$ \\
\hline Unimed & 0,3155 & 0,3117 & 0,1906 & 0,0982 & 0,5388 & $68,43 \%$ & $68,47 \%$ & $212,20 \%$ & $217,03 \%$ \\
\hline MetLife Vida e Previdência & 0,7573 & 0,7482 & 0,2173 & 0,0687 & 0,2282 & $24,29 \%$ & $24,26 \%$ & $32,40 \%$ & $273,19 \%$ \\
\hline Alfa Previdência Vida & 0,1920 & 0,1920 & 0,0804 & 0,0938 & 0,2032 & $80,80 \%$ & $80,80 \%$ & $433,41 \%$ & $422,40 \%$ \\
\hline Mongeral & 1,0000 & 0,2630 & 0,4950 & 0,0950 & 0,0350 & & EFICIENTE \\
\hline Previsul & 1,0000 & 1,2000 & 0,5290 & 0,1240 & 0,0550 & & EFICIENTE \\
\hline
\end{tabular}

Além disso, uma informação adicional pode ser encontrada no quadro anterior. Neste pode-se observar a necessidade de incremento que cada output de cada DMU ineficiente precisaria ter para que a mesma se tornasse eficiente. Percebe-se que as seguradoras de menor desempenho são aquelas que em média necessitam de maiores mudanças tanto nos níveis de inputs, quanto nos de outputs.

Uma outra análise interessante gerada pelo uso da DEA é a de benchmarking. Pode-se perceber que a Safra é a seguradora que mais aparece como benchmark para as outras em todas as análises. Isso se dá pelo fato de ser esta a seguradora de destaque, em termos de desempenho multicriterial, em relação às variáveis estudadas no período analisado.

Por último, ainda com base nos resultados da Tabela 04, procedeu-se duas análises sobre os resultados de 2005 em relação às variáveis. A primeira foi dos pesos atribuídos aos vetores de desempenho, para saber com base em pesos iguais a zero as variáveis que estavam sendo "desprezadas" na análise de desempenho. A segunda diz respeito a uma análise da necessidade média de melhoria nas variáveis. Para isso, 
Marcelo Alvaro da Silva Macedo, Ana Carolina Thomaz de Almeida Monteiro Barbosa

observou-se o quanto cada variável precisava melhorar em cada DMU não eficiente de modo a torná-la eficiente.

Destas duas análises pode-se identificar, então, quais eram as variáveis problemáticas, ou seja, as que mais apareciam com pesos zero e as que mais necessitavam de redução para chegar ao valor de referência. Ambas as análises apontaram para as mesmas variáveis, sinistralidade, como input, e margem, como, output.

\section{CONSIDERAÇÕES}

A eficiência é um dos aspectos que vêm governando, em maior ou menor grau, dependendo da organização, a atuação de seguradoras e outras instituições financeiras no Brasil e no mundo, nos últimos anos, conforme mostram os resultados dos estudos revelados nesta pesquisa.

Numa análise dos resultados apresentados, pode-se perceber que as melhores seguradoras são aquelas que possuem um desempenho multicriterial superior. Isto quer dizer que a seguradora Safra, que possui desempenho relativo superior, pode ser utilizada como benchmark para as demais. Por outro lado, seguradoras como Alfa e HSBC que têm, em geral, baixos índices de desempenho, precisam de mudanças drásticas em seus inputs e/ou outputs.

Uma situação que chama atenção é o desempenho das seguradoras Previsul e Itaú. A Previsul é financeiramente eficiente, mas operacionalmente muito ineficiente. Já a Itaú tem desempenho operacional máximo, mas possui eficiência financeira entre as piores da amostra.

No caso da Previsul, este resultado está em consonância com a constatação que as seguradoras têm seu resultado mantido, basicamente, em função de sua performance financeira. Isso é corroborado pelo fato de que a maioria das seguradoras possui desempenho melhor no modelo financeiro do que no modelo operacional. Já no caso do Itaú o resultado mostra uma tendência, abordada no trabalho de Macedo et al., (2006), de que as seguradoras estariam se voltando para melhorias operacionais. 
Marcelo Alvaro da Silva Macedo, Ana Carolina Thomaz de Almeida Monteiro Barbosa

Percebeu-se que neste segmento de seguros, as líderes em desempenho não eram as maiores instituições, nem em termos de receita, nem em termos de volume de aplicações. Além disso, observou-se que estas não estavam relacionadas a grandes conglomerados financeiros. A exceção foi a seguradora Itaú Vida.

Os resultados deste estudo revelam, como melhores seguradoras as de porte intermediário, comparadas com as demais da amostra. Isso mostra que as instituições de pequeno e médio porte possuem totais condições de sobrevivência e de competição, pois não é o tamanho que gera a competitividade, mas sim o desempenho superior.

Os resultados ainda propõem uma nova percepção sobre a performance financeira de seguradoras que não se encontram disponíveis aos gestores e ao mercado em geral, através dos balanços e tradicionais análises de índices financeiros. Ou seja, a partir de informações que não estariam disponíveis pelas técnicas convencionais, os resultados da análise envoltória de dados podem proporcionar melhores condições de competitividade às seguradoras, principalmente quando interpretadas e usadas com os conhecimentos e julgamentos próprios da alta administração sobre suas operações.

De maneira geral, as conclusões deste estudo estão em consonância com os resultados de Macedo et al., (2006) e contrários aos de Contador et al., (2000), Silva; Neves e Gonçalves Neto (2003) e Silva e Neves (2004), no que diz respeito a relação entre tamanho e desempenho e a ligação com conglomerados financeiros.

Assim como neste estudo, Macedo et al., (2006) concluíram que seguradoras de porte menor e não ligadas aos grandes conglomerados financeiros poderiam obter desempenho superior. Isso pode estar sinalizando para uma mudança de postura competitiva neste setor. Como as expectativas ao longo dos anos norteiam a diminuição da taxa de juros, se torna indispensável que as seguradoras busquem opções para reduzir a dependência do resultado financeiro, o que reforça a tese de que um melhor desempenho operacional é o que levará as seguradoras a obter ganhos. Neste sentido, até a exceção nos resultados confirma o exposto, já que a Itaú Vida, única seguradora de grande porte ligada a um grande conglomerado financeiro que obteve desempenho máximo, teve sua eficiência alcançada no modelo operacional. Ou seja, isso pode estar representando um indício de que até as seguradoras ligadas aos grandes conglomerados 
Desempenho organizacional de seguradoras de vida e previdência no Brasil: uma análise apoiada

Marcelo Alvaro da Silva Macedo, Ana Carolina Thomaz de Almeida Monteiro Barbosa

possam estar se voltando para obtenção de ganhos operacionais ao invés de resultados meramente financeiros.

Há muito a ser explorado desta metodologia em gestão de instituições financeiras. Este mesmo estudo pode ter continuidade através de uma melhor visão dos vetores de desempenho que mais contribuem para uma mensuração mais apurada da eficiência organizacional, aplicando a metodologia aqui apresentada e discutida, a outros segmentos de seguros no Brasil; combinando a metodologia DEA a outras metodologias como a análise discriminante.

\section{REFERÊNCIAS BIBLIOGRÁFICAS}

ARRIETA, A. e RIECKHOF, P. 2001. Es Beneficiosa la Especialización en Seguros de Vida en América Latina? Documentos de Trabajo - Superintendencia de Banca, Seguros y AFP del Perú. $17 \mathrm{p}$.

BANKER, R.D.; CHARNES, A. e COOPER, W.W. 1984. Some Models for Estimating Technical and Scale Inefficiencies in Data Envelopment Analysis. Management Science, 30(9):1078-1092.

CAMP, R.C. 1989. Benchm arking: the search for industry best practices that lead to superior performance. New York, Quality Resources, 299 p.

CHARNES, A.; COOPER, W. W. e RHODES, E. 1978. Measuring the Efficiency of Decision Making Units. European Journal of Operational Research, 2(6):429-444.

CHARNES, A.; COOPER, W.W.; LEWIN, A.Y. e SEIFORD, L. 1994. M. Data Envelopment Analysis: Theory, Methodology and Application. $2^{\mathrm{a}}$ ed., Boston, KAP, $513 p$.

COELLI, T.; RAO, D.S.P. e BALTESE, G.E. 1998. An Introduction to Efficiency and Productivity Analysis. Boston, KAP, $275 \mathrm{p}$.

CONTADOR, C.R.; et al. 2000. Avaliação da Performance do Mercado Segurador Brasileiro através do método DEA (Data Envelopment Analysis) no primeiro semestre de 1999. In: Simpósio Brasileiro de Pesquisa Operacional, 32, 2000, Viçosa/MG. SOBRAPO.

HSIAO, S.H. e SU, S.H. 2006. An Evaluation of Investment Performance and Financial Standing for Life Insuers in Taiwan. Journal of American Academy of Business, 
Desempenho organizacional de seguradoras de vida e previdência no Brasil: uma análise apoiada

10(1):278-284 .

LINS, M.P. E. e MEZA, L.Â. 2000. Análise Envoltória de Dados e Perspectivas de Integração no Ambiente de Apoio à Decisão. Rio de Janeiro, COPPE/UFRJ, 232 p.

MACEDO, M.A.S.; SILVA, F.F. e SANTOS, R.M. 2006. Análise do Mercado de Seguros no Brasil: uma visão do desempenho organizacional das seguradoras no ano de 2003. Revista Contabilidade e Finanças. Edição Especial - Atuaria, p. 88-100.

MAGALHÃES DA SILVA, A. C.; NEVES, C.; GONÇALVES NETO, A. C. 2003. Avaliação da Eficiência das Companhias de Seguro no ano de 2002: uma abordagem através da Análise Envoltória de Dados. In: CONGRESSO BRASILEIRO DE CUSTOS, 10, Guarapari/ES. 2003. Anais do X Congresso Brasileiro de Custos. Guarapari/ES, 2003. 1 CD.

MAGALHÃES DA SILVA, A. C.; NEVES, C. 2004. Um Estudo Exploratório da Estratégia do Canal de Distribuição das Seguradoras de Grande e Médio Porte do Brasil, através de um Modelo de Eficiência de suas Atividades no biênio 2002-2003. I n: CONGRESSO LATINO-AMERICANO DE ESTRATÉGIA, XVII, 2004, Itapema/SC. Anais do XVI I Congresso da SLADE. Itapema, 2004. 1 CD.

MEZA, L.A.; BIONDI NETO, L; SOARES DE MELLO, J.C.C.B.; GOMES. E.G. e COELHO, P.H.G. 2003.

SIAD - Sistema Integrado de Apoio à Decisão: uma implementação computacional de modelos de análise de envoltória de dados. I n: SIMPÓSIO DE PESQUISA OPERACIONAL DA MARINHA, 6, 2003, Rio de Janeiro. Anais do VI SPOLM. Rio de Janeiro, CASNAV, 2003. 1 CD.

NEELY, A. 1998. Measuring business performance. London, The Economist Books, 224vp.

NOVAES, A. G. 2004. Logística e Gerenciamento da Cadeia de Distribuição. Rio de Janeiro, Campus, 500 p. REVISTA VALOR FINANCEIRO. São Paulo: Valor Econômico, 2006. Anual. Os Maiores e Mais Eficientes.

SLACK, N.; CHAMBERS, S.; HARLAND, C.; HARRISON, A. e JOHNSTON, R. 2002. Administração da Produção. $2^{\mathrm{a}}$ ed. São Paulo, Atlas, 754 p.

VERGARA, S. C. 2007. Projetos e Relatórios de Pesquisa em Administração. 9ª ed. São Paulo, Atlas, 200 p.

YANG, Z. 2006. A two- stage DEA model to evaluate the overall performance of Canadian life and health insurance companies. Mathematical and Computer Modelling, 43:910919. 
Desempenho organizacional de seguradoras de vida e previdência no Brasil: uma análise apoiada em DEA Marcelo Alvaro da Silva Macedo, Ana Carolina Thomaz de Almeida Monteiro Barbosa

ZHU, J. 2000. Multi-factor Performance Measure Model with Application to Fortune 500 Companies. European Journal of Operational Research. 123 (1): 105-124.

Data de Submissão: 14/12/2007

Data de Aceite: 15/12/2007 